\title{
Moluscos terrestres de las elevaciones cársticas de Viñales, Pinar del Río, Cuba
}

\author{
Wilfredo Oliva-Olivera ${ }^{1,2} \&$ Raimundo Real ${ }^{2}$ \\ 1. Instituto de Ecología y Sistemática, Ciudad de la Habana, Cuba; oliva_olivera@hotmail.com \\ 2. Universidad de Málaga, Málaga, España; rrgimenez@uma.es
}

Recibido 05-VI-2008. Corregido 22-II-2009. Aceptado 25-III-2009.

\begin{abstract}
Land snails from the Viñales limestone highlands, Pinar del Río, Cuba. The land snails inhabiting the limestone highlands in Cuba are poorly known. From field surveys, traditional collection catalogues, and the literature, we list 136 species, 49 genera and 19 families, $90 \%$ of the species endemic to Cuba; only five species are non-indigenous of the Cuban archipelago. Annulariidae and Urocoptidae are the most represented families, and Chondrothyra and Liocallonia are the genera with the highest number of species. Forty-four species of this list were absent from the consulted collections. We provide 16 new records of species in localities of the region; and 12 species seem to have become locally extinct. Rev. Biol. Trop. 57 (3): 589-604. Epub 2009 September 30 .
\end{abstract}

Key words: gastropods, malacological collections, species diversity, distribution, Viñales, Cuba.

Los moluscos constituyen el segundo filo con mayor número de especies animales, superado sólo por los artrópodos (Ruppert \& Barnes 1996). Se han registrado 30 mil especies de moluscos terrestres, todos gasterópodos, distribuidos ampliamente por el mundo (Abbott 1989). El archipiélago cubano cuenta con 1299 de estas especies de gasterópodos, agrupadas en 158 géneros y 32 familias (Espinosa \& Ortea 1999), y posiblemente sea una de las áreas con mayor densidad de especies de moluscos terrestres del mundo (Torre \& Bartsch 1938). Este grupo zoológico constituye uno de los de mayor diversidad y porcentaje de endemismo de Cuba (Vales et al. 1998) y se distribuye a través de los principales sistemas montañosos del archipiélago, representados por las cordilleras Nipe-Sagua-Baracoa, Guamuhaya y Guanigüanico (Espinosa et al.1994, Espinosa \& Ortea 1999).

Las publicaciones sobre comunidades de moluscos terrestres de Cuba son escasas (Pérez et al.1996). En general, los moluscos terrestres constituyen un grupo poco estudiado en los bosques tropicales (Winter \& Gittenberger 1998) y aunque su riqueza de especies tiende a ser alta, resulta difícil de describir a causa de la baja densidad poblacional y del tamaño pequeño de sus conchas (Emberton et al.1996). Como consecuencia, sus patrones de diversidad y endemismo son poco conocidos (Emberton et al.1997) y existe poca información comparable sobre los mismos (Tattersfield 1996).

Por otra parte, los sistemas montañosos son susceptibles a la erosión acelerada de los suelos, los desprendimientos de tierra y al rápido empobrecimiento de la diversidad genética (Programa 21, 1992). De acuerdo a la Estrategia Nacional para la Diversidad Biológica y Plan de Acción en la República de Cuba (Vilamajó et al.2002), es necesario priorizar el monitoreo de áreas críticas, como los sistemas montañosos, y completar los vacíos de conocimiento identificados en el Estudio Nacional sobre 
la Diversidad Biológica. Además, el declive que está teniendo lugar en las poblaciones de moluscos terrestres del mundo (Lydeard et al.2004) demanda estudios básicos orientados al conocimiento de la composición, estructura y funcionamiento de sus comunidades, sobre cuyas bases desarrollar planes de manejos orientados a su protección y conservación.

En esta publicación nos hemos propuesto: a) describir la composición de especies de moluscos terrestres de Viñales, una de las regiones malacológicamente más diversas del archipiélago cubano (Espinosa \& Ortea 1999) y declarada parque nacional en el 2001 (Landeiro 2003); b) informar sobre la ampliación de distribución para algunas especies cuyo registro de localidades, según la literatura, aún no había sido incluido en áreas de Viñales; c) detectar la presencia de especies de moluscos terrestres sinantrópicas como indicadoras de antropización; d) alertar sobre posibles extinciones.

\section{MATERIALES Y MÉTODOS}

Área de estudio: La región de Viñales se localiza en el municipio del mismo nombre, ubicado en la porción centro norte de la provincia cubana de Pinar del Río. Es una de las regiones geoecológicas, junto con Guane, Sumidero y la Güira, de las que está compuesta las Alturas y Montañas de los Órganos (Luis 2000), uno de los sistemas montañosos, que, unido al del Rosario, conforman la cordillera de Guanigüanico.

Aunque su relieve incluye tanto alturas de pizarras como elevaciones cársticas, la mayoría de las especies de moluscos terrestres de Viñales limitan su distribución a las elevaciones cársticas. La naturaleza cárstica de estas elevaciones las hace susceptible a la acción disolvente del agua, mediante cuya influencia se han originado una diversidad de rasgos geomorfológicos del tipo cárstico y de torres (Núñez et al.1984). A partir de la caracterización paisajística desarrollada por Luis (2000), se puede decir que sus elevaciones están representadas actualmente por tres tipos de localidades: carso ruiniforme, mogotes aislados y sierras calcáreas.
Según Luis (2000), las sierras calcáreas presentan áreas mayores que un kilómetro cuadrado; incluyen todas las variedades posibles de formas del micro relieve cárstico representadas por paredones, cimas, hoyos, taludes y abras; su cobertura pedológica es abundante; el comportamiento climático es complejo y en ellas crecen todas las formaciones vegetales presentes en el área de estudio. Los mogotes aislados poseen áreas menores que un kilómetro cuadrado; incluyen solamente dos o tres variedades de formas del micro relieve representadas por paredones, cimas, y en algunos casos taludes; su cobertura pedológica es escasa, el comportamiento climático no es tan complejo como el de las sierras, y en ellos no crecen todas las formaciones vegetales presentes en el área de estudio. Los carsos ruiniformes generalmente presentan superficies menores que 0.05 kilómetros cuadrados; carecen de formas del micro relieve cárstico definidas como las de las sierras y mogotes; presentan poca cobertura pedológica, poca complejidad climática y elementos de todas las formaciones vegetales que se encuentran en el área de estudio.

La mayoría de las localidades a las que se hace referencia durante el estudio están incluidas en los límites actuales del Parque Nacional Viñales. La composición taxonómica de moluscos terrestres de la región se ha determinado a partir de los muestreos efectuados durante cuatro años en localidades representativas de la región; la consulta de la literatura; y la revisión de los catálogos no automatizados de las colecciones malacológicas de referencia nacional depositadas en el Instituto de Ecología y Sistemática de Cuba.

Muestreos: Los muestreos desarrollados durante el presente estudio se dirigieron a 12 localidades representativas de las elevaciones cársticas de Viñales, e incluyen la única localidad, Sierra del Medio, representante de carso ruiniforme presente en Viñales (Cuadro 1). Estos muestreos se llevaron a cabo desde 2002 hasta 2005 durante junio, julio y agosto. Cada localidad se muestreó en un solo año y en ella se revisaron 100 sitios distribuidos equitativamente 
CUADRO 1

Localización en grados $(G)$, minutos $(M)$, y segundos $(S)$ de acuerdo al sistema de proyección WGS 84 de las localidades representativas seleccionadas para muestrear

TABLE 1

Localization (degrees) $(G)$, minutes $(M)$, seconds $(S)$ according to the WGS 84 projection system of representative localities selected for sampling

$\begin{array}{lccc}\text { Localidades } & \text { Tipo de Localidad } & \text { Longitud. GMS } & \text { Latitud. GMS } \\ \text { M. El Pájaro } & \text { Mogote Aislado } & -83,41,31.4844 & 22,49,3.9684 \\ \text { M. La Feita } & \text { Mogote Aislado } & -83,43,24.1392 & 22,36,1.1844 \\ \text { M. Coco Solo } & \text { Mogote Aislado } & -83,41,46.5324 & 22,37,33.8592 \\ \text { M. Zacarías } & \text { Mogote Aislado } & -83,41,5.6652 & 22,39,25.4844 \\ \text { M. Irene } & \text { Mogote Aislado } & -83,42,50.6268 & 22,38,21.7536 \\ \text { M. Pan de Azúcar } & \text { Mogote Aislado } & -83,50,12.5376 & 22,37,44.8932 \\ \text { S. Tumbadero } & \text { Sierra Calcárea } & -83,43,32.6352 & 22,37,20.1972 \\ \text { S. Infierno-Viñales } & \text { Sierra Calcárea } & -83,46,56.676 & 22,37,51.114 \\ \text { S. Guasasa } & \text { Sierra Calcárea } & -83,42,8.5788 & 22,39,46.0944 \\ \text { S. Fermín-Chichones } & \text { Sierra Calcárea } & -83,52,10.1604 & 22,35,18.4452 \\ \text { S. Quemado } & \text { Sierra Calcárea } & -83,51,13.8132 & 22,31,52.7268 \\ \text { S. La Caoba } & \text { Carso Ruiniforme } & -83,51,31.446 & 22,33,45.54\end{array}$

entre sus formas de micro relieve cárstico; los sitios dentro de cada una de estas formas de micro relieve fueron seleccionados al azar. En los diferentes sitios se revisó por inspección visual una parcela de vegetación de $3 \times 3 \mathrm{~m}$ (y hasta $3 \mathrm{~m}$ de altura), una parcela de superficie de suelo de $1 \times 1 \mathrm{~m}$ y una parcela de suelo de $0.25 \times 0.25 \times 0.05 \mathrm{~m}$ de profundidad, para detectar los moluscos arborícolas, terrícolas-petrícolas y micro moluscos respectivamente. Las muestras de hojarasca y de cubierta pedológica tomadas de cada sitio, según su disponibilidad y hasta cinco centímetros de profundidad, se trasladaron hacia el laboratorio en bolsas de plástico; se hicieron pasar por tamices de $9.52 \mathrm{~mm}$ y $3.15 \mathrm{~mm}$; y se revisaron a simple vista durante la tarde del día de muestreo. En total se revisaron 300 parcelas en cada una de las doce localidades muestreadas, en un horario comprendido entre las 8:00 h y las $12: 00 \mathrm{~h}$ de los días de muestreo destinados a cada localidad.

El material recolectado se depositó en las Colecciones del Instituto de Ecología y Sistemática del Ministerio de Ciencia y Tecnología y Ambiente de Cuba.
Revisión de los catálogos de colecciones: También se consultaron los catálogos no automatizados de la colección malacológica depositada en el Instituto de Ecología y Sistemática, que cuenta con 750 mil ejemplares y está dividida en Colección Histórica, Müller y Sánchez de Fuentes, y Colección Básica que se incrementa progresivamente con el aporte de los investigadores, técnicos, donaciones e intercambios. La revisión del catálogo no automatizado correspondiente a la Colección Básica se limitó a las familias Urocoptidae, Annularidae y Pomatiasidae, las de mayor riqueza de especies en la malacofauna cubana. Todas las especies de moluscos terrestres presentes en dichas colecciones están representadas por conchas de sus correspondientes ejemplares adultos.

Revisión bibliográfica: La revisión de la literatura se limitó al catálogo más reciente para los moluscos terrestres del archipiélago cubano (Espinosa \& Ortea 1999), pues el resto de la información permanece dispersa, desactualizada (Espinosa et al.1994) y de difícil acceso. Además, en el ámbito de la malacología cubana 
han prevalecido los estudios poblacionales, las publicaciones sobre comunidades de moluscos terrestres son escasas y en general no tienen en cuenta a los micros moluscos presentes en las áreas estudiadas.

Durante la consulta del catálogo hemos excluido deliberadamente las especies con distribución imprecisa, ambigua o generalizada, de tipo "por todo el archipiélago cubano" o por "todo Pinar del Río", a menos que presenten algún registro concreto en las colecciones o hayan sido observadas durante los muestreos. Tampoco hemos incluido las especies Cochlodinella nana, Teniustemma multispiralis, Tomelasmus denticulatus, Tomelasmus tumidiorus y Oleacina wrighti cuyas distribuciones son desconocidas.

\section{RESULTADOS}

Se encontró un total de 136 especies de moluscos terrestres, agrupadas en 49 géneros y
19 familias, pertenecientes a la región geoecológica de Viñales (Cuadro 2). El $90 \%$ de estas especies son endémicas, el $57 \%$ lo son nacionales, el $20 \%$ regionales y el $13 \%$ locales. Se observaron cinco especies no autóctonas del archipiélago y que según Espinosa \& Ortea (1999) han sido introducidas durante décadas anteriores en el país.

El género Chondrothyra es el que presenta mayor número de especies, con un total de 14 representantes, seguido por el género Liocallonia con 12 especies. Las familias Annulariidae y Urocoptidae son las mejores representadas con 36 y 34 especies, respectivamente.

De acuerdo a los muestreos efectuados durante este estudio y al catálogo de Espinosa \& Ortea (1999), existen 45 especies de moluscos terrestres distribuidas en las elevaciones cársticas de Viñales que hasta el momento no presentaban ejemplares de sus poblaciones depositados en las colecciones malacológicas

CUADRO 2

Composición taxonómica de los moluscos terrestres de las elevaciones cársticas de Viñales según tres fuentes

TABLE 2

Taxonomic composition of land snails from limestone highlands inf Viñales according to three sources

\begin{tabular}{|c|c|c|c|}
\hline Taxon & Muestreo & Colección & Literatura \\
\hline \multicolumn{4}{|l|}{ Helicinidae } \\
\hline Alcadia rotunda* (Orbigny, 1842) & $\mathrm{X}$ & $\mathrm{X}$ & $\mathrm{X}$ \\
\hline Alcadia dissimulans* (Poey, 1858) & $\mathrm{X}$ & $\mathrm{X}$ & $\mathrm{X}$ \\
\hline Alcadia hispida* (Pfeiffer, 1839) & & $\mathrm{X}$ & $\mathrm{X}$ \\
\hline Alcadia minima* (Orbigny, 1842) & $\mathrm{X}$ & $\mathrm{X}$ & $\mathrm{X}$ \\
\hline Emoda sagraiana* (Orbigny, 1842) & $\mathrm{X}$ & $X$ & $\mathrm{X}$ \\
\hline Troschelviana chrysochasma* (Poey, 1853) & $\mathrm{X}$ & $\mathrm{X}$ & $\mathrm{X}$ \\
\hline Troschelviana erythracea* (Wright in Sowerby, 1866) & & & $\mathrm{X}$ \\
\hline Troschelviana jugulata* (Poey, 1858) & & $\mathrm{X}$ & \\
\hline Troschelviana rubromarginata* (Gundlach in Poey, 1858) & $X$ & & $\mathrm{X}$ \\
\hline Helicina adspersa* (Pfeiffer, 1839) & $\mathrm{X}$ & $\mathrm{X}$ & $\mathrm{X}$ \\
\hline Semitrochatella alboviridis* (Wright in Pfeiffer, 1864) & & & $\mathrm{X}$ \\
\hline Semitrochatella elongata* (Orbigny, 1842) & $\mathrm{X}$ & $\mathrm{X}$ & $\mathrm{X}$ \\
\hline Semitrochatella fuscula* (Gundlach in Pfeiffer, 1863) & $\mathrm{X}$ & & $\mathrm{X}$ \\
\hline Ustronia acuminata* (Velásquez in Poey, 1852) & $\mathrm{X}$ & $\mathrm{X}$ & $\mathrm{X}$ \\
\hline Viana regina* (Morelet, 1849) & $\mathrm{X}$ & $\mathrm{X}$ & $\mathrm{X}$ \\
\hline
\end{tabular}


CUADRO 2 (Continuación)

Composición taxonómica de los moluscos terrestres de las elevaciones cársticas de Viñales según tres fuentes

TABLE 2 (Continued)

Taxonomic composition of land snails from limestone highlands inf Vinales according to three sources

\section{Taxon}

Proserpinidae

Proserpina depresa* (Orbigny, 1842)

Proserpina globulosa* (Orbigny, 1842)

Megalosmastomidae

Farcimen hendersoni* (Torre \& Bartsch, 1942)

Farcimen mani* (Poey, 1851)

Farcimen superbum* (Torre \& Bartsch, 1942)

Farcimen vignalense* (Torre \& Bartsch, 1942)

Farcimen subventricosum* (Torre \& Bartsch, 1942)

Annulariidae

Annularops attenuata* (Torre \& Bartsch, 1941)

Annularops blaini* (Gundlach in Pfeiffer, 1863)

Annularops coronadoi* (Arango in Poey, 1867)

Annularops tryoni* (Arango, 1979)

Annularops semicana* (Morelet, 1851)

Annularop plicata*** (Torre \& Bartsch, 1941)

Blaesospira echinus** (Wright in Pfeiffer, 1864)

Chondropometes torrei* (Bartsch, 1937)

Chondropometes eximium* (Torre \& Bartsch, 1938)

Chondropometes saccharinum ** (Torre \& Bartsch, 1938)

Chondropometes segregatum* (Torre \& Bartsch, 1938)

Chondropometes vignalense $* *$ (Wright in Pfeiffer, 1863)

Rhytidothyra bilabiata* (Orbigny, 1842)

Chondrothyra affinis** (Torre \& Bartsch, 1938)

Chondrothyra cerina* (Torre \& Bartsch, 1938)

Chondrothyra gundlachi* (Arango, 1862)

Chondrothyra impresa* (Torre \& Bartsch, 1938)

Chondrothyra incrassata* (Wright in Pfeiffer, 1862)

Chondrothyra parilis* (Torre \& Bartsch, 1938)

Chondrothyr. reticulata* (Torre \& Bartsch, 1938)

Chondrothyra rutila** (Torre \& Bartsch, 1938)

Chondrothyra tosta** (Torre \& Bartsch, 1938)

Chondrothyra shuttleworthi* (Pfeiffer, 1851)

Chondrothyra egregia* (Gundlach in Pfeiffer, 1856)

Chondrothyra subgregia* (Torre \& Bartsch, 1938)

Chondrothyra tenebrata* (Torre \& Bartsch, 1938)

Muestreo Colección Literatura

X

$\mathrm{X}$

$\mathrm{X}$

X

X

$\begin{array}{ccc} & X & X \\ X & X & \\ X & X & X \\ X & X & X \\ & X & X\end{array}$

$\mathrm{X}$

X

X

X

$\mathrm{X}$

$\mathrm{X}$

$\mathrm{X}$

$\mathrm{X}$

X $\quad \mathrm{X}$

$\mathrm{X}$

$\mathrm{X}$

$\mathrm{X}$

$\mathrm{X}$

$\mathrm{X}$

$\mathrm{X}$

$\mathrm{X}$

$\mathrm{X}$

X

$\mathrm{X}$

$\mathrm{X}$

$\mathrm{X}$

$\mathrm{X}$

$\mathrm{X}$

$\mathrm{X}$

$\mathrm{X}$

$\mathrm{X}$

$\mathrm{X}$

$\mathrm{X}$

$\mathrm{X}$

$\mathrm{X}$

$\begin{array}{ll}\mathrm{X} & \mathrm{X}\end{array}$

X

$\mathrm{X}$

$\mathrm{X}$

$\mathrm{X}$

$\mathrm{X}$

X

$\mathrm{X}$

$\mathrm{X}$

X

X

X

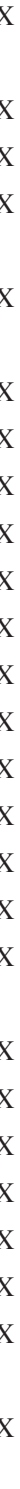


CUADRO 2 (Continuación)

Composición taxonómica de los moluscos terrestres de las elevaciones cársticas de Viñales según tres fuentes

TABLE 2 (Continued)

Taxonomic composition of land snails from limestone highlands inf Viñales according to three sources

Taxon
Chondrothyra uniplicata* (Torre \& Bartsch, 1938)

Eutudorops complanata*** (Torre \& Bartsch, 1941)

Eutudorops troschel $*$ (Pfeiffer, 1864)

Eutudorops undosa* (Gundlach in Pfeiffer, 1863)

Hendersonina hendersoni*** (Torre, 1909)

Hendersonina discolorans $*($ Morelet, 1849)

Hendersonina echinulata** (Wright in Pfeiffer, 1863)

Hendersonina hamlini* (Arango, 1882)

Hendersonina deceptor** (Arango, 1882)

Hendersonina mendaz *** (Torre \& Bartsch, 1938)

Hendersonina sinuosa* (Wright in Pfeiffer, 1862)

Truncatellidae

Truncatella sp.

Veronicellidae

Leidyula floridiana (Leidy, 1851)

Veronicella tenax* (Baker, 1931)

Orthalicidae

Liguus fasciatus* (Müller, 1774)

Liguus flammelus* (Clench, 1934)

Vertiginidae

Sterkia antillensis (Pilsbry, 1940)

Urocoptidae

Capillacea capillacea* (Pfeiffer, 1863)

Capillacea pulcherrima** (Jaume \& Torre, 1976)

Capillacea angustior** (Wright in Pfeiffer, 1864)

Gemnicoptis rocai** (Torre, 1929)

Gemnicoptis terebella*** (Torre, 1929)

Organocoptis constantia*** (Jaume \& Torre, 1976)

Cochlodinella atropurpurea* (Arango, 1882)

Cochlodinella atra*** (Jaume \& Torre, 1976)

Liocallonia attenuata*** (Jaume \& Torre, 1976)

Liocallonia cuestai $* * *$ (Torre, 1930)

Liocallonia oligomesus *** (Pilsbry, 1903)

Liocallonia palmae* (Gundlach in Pfeiffer, 1876)

Liocallonia propincua $* *$ (Gundlach in Arango, 1882)

Liocallonia doloris*** (Jaume \& Torre, 1976)

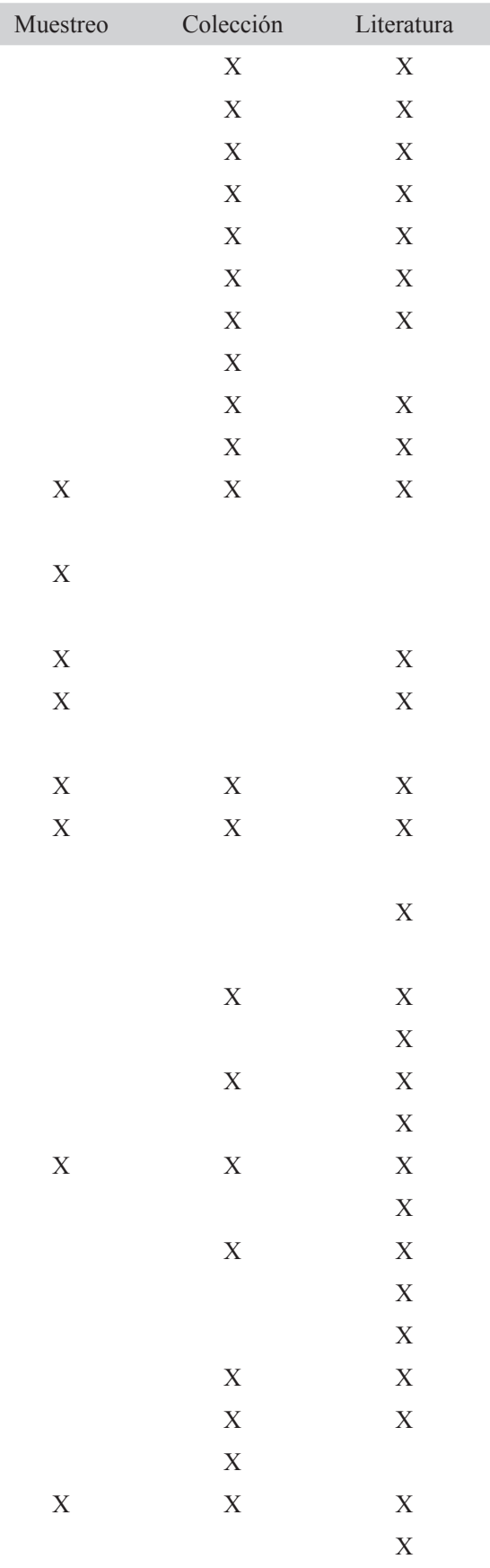


CUADRO 2 (Continuación)

Composición taxonómica de los moluscos terrestres de las elevaciones cársticas de Viñales según tres fuentes

TABLE 2 (Continued)

Taxonomic composition of land snails from limestone highlands inf Viñales according to three sources

Taxon $\quad$ Muestreo Colección Literatura

Liocallonia infortunata** (Arango, 1882)

$\mathrm{X}$

$\mathrm{X}$

Liocallonia andresensis* (Jaume \& Torre, 1976)

$\mathrm{X}$

Liocallonia canaletensis* (Jaume \& Torre, 1976)

$\mathrm{X}$

Liocallonia jaguaensis*** (Jaume \& Torre, 1976)

$\mathrm{X}$

Liocallonia minaensis*** (Jaume \& Torre, 1976)

$\mathrm{X}$

Liocallonia triplicata*** (Arango, 1882)

$\mathrm{X}$

Nodulia amoenivallis** (Pilsbry, 1929)

$\mathrm{X}$

Nodulia caponensis*** (Jaume \& Torre, 1976)

$\mathrm{X}$

Nodulia corpulenta** (Spence, 1936)

Nodulia nodulifera** (Torre, 1929)

Nodulia oblita*** (Jaume et Torre, 1976)

Nodulia vignalensis** (Wright in Pfeiffer, 1863)

Tomelasmus coloratus** (Arango, 1882)

Tomelasmus azucarensis*** (Jaume \& Torre, 1976)

$\mathrm{X}$

Tomelasmus hesperius* (Jaume \& Torre, 1976)

Tomelasmus irroratus* (Gundlach, 1856)

Tomelasmus semicoloratus* (Spence, 1936)

Tomelasmus thomsoni* (Arango, 1884)

Tomelasmus assimilis** (Arango, 1884)

Callocoptis hubbardi*** (Jaume \& Torre, 1976)

X

$\mathrm{X}$

$\mathrm{X}$

Microceramidae

Microceramus elegans* (Gundlach in Pfeiffer, 1863)

X

Microceramus latus* (Gundlach in Pfeiffer, 1863)

Microceramus costellaris** (Gundlach in Pfeiffer, 1863)

Microceramus perconicus * (Pilsbry)

Microceramus infradenticulatus* (Wright in Pfeiffer, 1864)

Microceramus paivanus* (Pfeiffer, 1866)

$\mathrm{X}$

$\mathrm{X}$

$\mathrm{X}$

$\mathrm{X}$

$\mathrm{X} \quad \mathrm{X}$

$\mathrm{X}$

$\mathrm{X}$

$\mathrm{X}$

$\mathrm{X}$

Subulinidae

Subulina octona (Bruguére, 1792)

Opeas pumilum (Pfeiffer, 1840)

Obeliscus gundlachi* (Pfeiffer, 1863)

X

$\mathrm{X}$

$X \quad X$

$\mathrm{X} \quad \mathrm{X}$

$\mathrm{X} \quad \mathrm{X}$

$\mathrm{X}$

$\mathrm{X}$

Leptinaria sp.

Pseudosubulina exilis (Pfeiffer, 1839)

$X \quad X$

X

$\mathrm{X}$

$\mathrm{X}$

$\mathrm{X}$

$\mathrm{X}$

X

$\mathrm{X}$

Oleacinidae

Oleacina incisa * (Pfeiffer, 1867)

Oleacina oleacea* (Férussac)

$\begin{array}{lll} & X & X \\ X & X & X\end{array}$

X

X $\quad X$

$\mathrm{X}$

$\mathrm{X}$

$\mathrm{X}$

$\mathrm{X}$

$\mathrm{X}$

$\mathrm{X}$ 
CUADRO 2 (Continuación)

Composición taxonómica de los moluscos terrestres de las elevaciones cársticas de Viñales según tres fuentes

TABLE 2 (Continued)

Taxonomic composition of land snails from limestone highlands inf Viñales according to three sources

$\begin{array}{lr}\text { Taxon } & \text { Muestrina ottonis* (Pfeiffer) } \\ \text { Oleacinacinidae } & \text { Xleacina solidula* (Pfeiffer, 1840) } \\ \text { Oleacina straminea* (Deshayes, 1819) } & \text { X } \\ \text { Oleacina subulata* (Pfeiffer, 1839) } & \mathrm{X} \\ \text { Rectoleacina cubensis* (Orbigny, 1842) } & \mathrm{X} \\ \text { Melaniella acuticostata* (Orbigny, 1842) } & \mathrm{X}\end{array}$

Streptaxidae

Streptostele musaecola (Morelet, 1860)

$\begin{array}{lrr}\text { X } & \text { Colección } & \text { Liter } \\ \mathrm{X} & \mathrm{X} & \mathrm{X} \\ \mathrm{X} & \mathrm{X} & \mathrm{X} \\ \mathrm{X} & & \mathrm{X} \\ \mathrm{X} & & \mathrm{X} \\ \mathrm{X} & \mathrm{X} & \mathrm{X}\end{array}$

Succinidae

Succinea ochracina* (Gundlach, 1858)

Succinea sagra* (Orbigny, 1842)

X

X

Sagdidae

Hojeda boothiana* (Pfeiffer, 1839)

Lacteoluna selenina* (Gould, 1839)

X

$\begin{array}{ll}X & X\end{array}$

Volvidens tichostoma* (Pfeiffer, 1839)

Vitrinidae

Hawaiia minuscula (Binney, 1840)

$\mathrm{X}$

Hawaiia apex** (C. B. Adams, 1849)

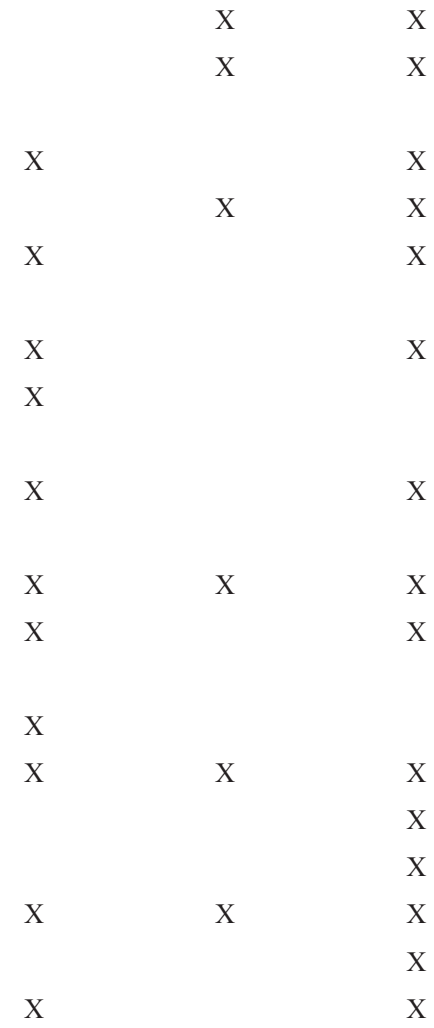

Bradybaenanidae

Bradybaena similares (Férussac, 1821)

X

Camaenidae

Zachrysia guanensis* (Poey, 1857)

Zachrysia auricoma (Férussac, 1821)

X

Helminthoglyptidae

Eurycampta pinarensis* (Aguayo)

Jeanneretia parraiana* (Orbigny, 1842)

Jeanneretia gundlachi** (Clench \& Aguayo, 1951)

*especie endémica nacional; **especie endémica regional; ***especie endémica local.

*national endemic species; **regional endemic species; local endemic species. 
(Cuadro 3). A través de este trabajo se han ampliado las colecciones zoológicas de referencia nacional con el aporte, al departamento de colecciones del IES, de ejemplares de 23 de estas especies observadas durante los muestreos, lo que resulta relevante teniendo en cuenta que las colecciones zoológicas, en general, funcionan como un centro de documentación en el que se almacena información potencialmente utilizable en estudios ecológicos y biogeográficos sobre los animales preservados en ellas. También se facilita la localización de las otras 22 especies que faltan por incorporar a las colecciones (Cuadro 3), y que aparecen citadas en el catálogo de Espinosa \& Ortea (1999), para que se tengan en cuenta durante estudios posteriores y en el desarrollo de colecciones malacológicas de referencia nacional.

\section{CUADRO 3}

Especies de moluscos terrestres de las elevaciones cársticas de Viñales no representadas en las colecciones malacológicas del IES

TABLE 3

Land snail species from the limestone highlands of Vinales not represented in the malacological collections of the IES

\begin{tabular}{|c|c|}
\hline Especie & Distribución \\
\hline Troschelviana erythracea & Cayos de San Felipe; Cayos de San Vicente \\
\hline Troschelviana rubromarginata* & Cayos de San Felipe; Sierra Quemada \\
\hline Semitrochatella alboviridis & Dos Hermanos \\
\hline Semitrochatella fuscula* & Pan de Azúcar; Mogote Dopico; Sierra Tumbadero \\
\hline Chondropometes eximium & Sierra del Quemado \\
\hline Chondropometes saccharinum* & Sierra Pan de Azúcar; Sierra del Martillo \\
\hline Trunchatella sp. * & Mogote La Feita \\
\hline Leidyula floridiana* & Mogote Dopico \\
\hline Veronicella tenax* & En todas las localidades muestreadas \\
\hline Sterkia antillensis & Localidades de Viñales no precisadas en el catálogo \\
\hline Anunularops alicata & Mogote de la Jagua \\
\hline Chochlodinella atra & Hoyo de San Antonio, al este del asiento poblacional de la Jagua \\
\hline Capillacea pulcherrima & $\begin{array}{l}\text { Segundo mogote al oeste del kilómetro } 14 \text { de la carretera entre Viñales y Pinar del } \\
\text { Río }\end{array}$ \\
\hline Geminicoptis rocai & Ensenada de San Vicente, Laguna de Piedras \\
\hline Liocallonia andresensis & Hoyo de San Antonio \\
\hline Liocallonia canaletensis & Mogote Rinconada, cerca del mogote La Mina \\
\hline Liocallonia jaguaensis & La Jagua de Azcui \\
\hline Liocallonia minaensis & Mogote La Mina \\
\hline Liocallonia triplicata & La Jagua de Azcui \\
\hline Liocallonia attenuata & Cerca del kilómetro 14 de la carretera entre Viñales y Pinar del Río \\
\hline Liocallonia doloris & Paso Dolores, Oeste del kilómetro 14 de la carretera entre Viñales y Pinar del Río \\
\hline Organocoptis constantia & Mogote de La Mina \\
\hline Nodulia caponensis & Mogote Capón \\
\hline Tomelasmus hesperius & Sierra de Viñales \\
\hline Tomelasmus azucarensis & Pan de Azúcar \\
\hline Microceramus elegans* & Pan de Azúcar; Sierra Guasasa \\
\hline Opeas pumilum* & En todas las localidades muestreadas, excepto los mogotes Coco Solo e Irene \\
\hline Obeliscus gundlachi* & En todas las localidades muestreadas \\
\hline Leptinaria sp. * & Sierra Pan de Azúcar \\
\hline
\end{tabular}


CUADRO 3 (Continuación)

Especies de moluscos terrestres de las elevaciones cársticas de Viñales no representadas

en las colecciones malacológicas del IES

TABLE 3 (Continued)

Land snail species from the limestone highlands of Viñales not represented in the malacological collections of the IES

\begin{tabular}{|c|c|}
\hline Especie & Distribución \\
\hline Pseudosubulina exilis* & Mogotes Zacarías; Coco Solo; Sierras Viñales Pan de Azúcar; La Caoba; Guasasa \\
\hline Oleacina ottonis* & En todas las localidades muestreadas, excepto Sierra Guasasa \\
\hline Oleacina subulata* & $\begin{array}{l}\text { Mogotes La Feita; Zacarías; Dopico ; Sierras Pan de Azúcar; Viñales; Tumbadero; } \\
\text { Guasasa }\end{array}$ \\
\hline Rectoleacina cubensis* & $\begin{array}{l}\text { Mogotes Pan de Azúcar; Irene; Sierras Quemada; Viñales; Guasasa; Tumbadero; } \\
\text { Pan de Azúcar }\end{array}$ \\
\hline Streptostele musaecola* & Sierra Guasasa \\
\hline Volvidens tichostoma* & Mogote Zacarías \\
\hline Hawaiia minuscula* & En todas las localidades muestreadas, excepto Mogotes Pan de Azúcar y Dopico. \\
\hline Hawaiia apex* & En todas las localidades muestreadas \\
\hline Zachrysia auricoma* & Mogotes La Feita; Coco Solo \\
\hline Bradybaena similaris* & Sierras Viñales; Guasasa; Tumbadero \\
\hline Eurycampta pinarensis* & $\begin{array}{l}\text { En todas las localidades muestreadas, excepto los Mogotes Zacarías, La Feita; } \\
\text { Dopico }\end{array}$ \\
\hline Jeanneretia gundlachi & Mogote La Resbalosa, Laguna de Piedra; Costanera de San Vicente \\
\hline Jeanneretia modica & Localidades de Viñales no precisadas en el catálogo \\
\hline Jeanneretia wrighti & Mogote la Iglesia \\
\hline Setipellis stigmatica* & En todas las localidades muestreadas, excepto Mogotes Dopico y Pan de Azúcar \\
\hline Hojeda bothiana* & $\begin{array}{l}\text { Mogotes Pan de Azúcar; Zacarías; Coco Solo; Sierras La Caoba; Quemada; } \\
\text { Tumbadero; Pan de Azúcar }\end{array}$ \\
\hline
\end{tabular}

Por otra parte, según los muestreos y los catálogo de colecciones consultados, existen 16 especies de moluscos terrestres que la literatura (fundamentalmente Espinosa et al.1994, Espinosa y Ortea 1999) aún no había registrado en las elevaciones cársticas de Viñales y por consiguiente son nuevos registros de localidades (Cuadro 4). En este cuadro también se incluyen cinco especies que, de acuerdo a la literatura, habían sido detectadas solamente en unas pocas localidades de Viñales, pero que los muestreos efectuados durante este estudio han revelado que están presentes en

otras localidades, evidenciando más bien una distribución generalizada por toda la región de elevaciones cársticas de Viñales.

A pesar de que el promedio de distancia mínima entre las localidades representativas de las elevaciones cársticas de Viñales seleccionadas para muestrear fue de $8.25 \mathrm{~km}$, solo se observaron 69 especies durante los muestreos. Sin embargo, el número total de ejemplares vivos identificados en la revisión de las parcelas fue de 14922 .

En este estudio se han detectado 12 especies (Cuadro 5) que de acuerdo a la literatura 
CUADRO 4

Nuevos registro

TABLE 4

New records

$\begin{array}{lll}\text { Especie } & \text { Distribución conocida } & \text { Distribución ampliada } \\ \text { Troschelviana jugulata } & \begin{array}{l}\text { Sierra de Guane; Sumidero, La Muralla; Los } \\ \text { Portales y otras localidades de la región de } \\ \text { Guane en Pinar del Río. }\end{array} & \text { Numerosas localidades de Viñales } \\ & \text { Sierra de Rangel en La Palma; } & \begin{array}{l}\text { Sierras Tumbadero, Derrumbada, Dos } \\ \text { Farcimen mani }\end{array} \\ & \begin{array}{l}\text { Hermanas; Quemada; Viñales; Mogote } \\ \text { Zacarías; Dopico; }\end{array}\end{array}$

Annularops blaini

Chondrothyra gundlachi

Chondrothyra egregia

Chondrothyra tenebrata

Hendersonina hamlini

Truncatella sp.

Liocallonia palmae

Proserpina globulosa

Microceramus perconicus

Microceramus paivanus

Leptinaria sp.

Pseudosubulina exilis

Hawaiia apex
Sierra de la Guira y mogotes adyacentes; La Cumbre, cerca de San Diego de los Baños

Sierra Paso Real en Guane; Sierra de Guane; Puerta de la Muralla; Los Portales

Hato de Caimito, al oeste del Pan de Guajaibón

Pasada de las Escaleras en Sierra San Andrés; Sierra Guacamayas; Ensenada de la Ayúa; Mogote del Canalete; Mogote Largo al suroeste de la Sierra Guacamayas.

Mogote Cabrera y de la Vega, entre Pinar del Río y Luis Lazo

Distribución Costera

Entre La Palma y Caiguanabo.

No registrada en el catálogo

Distribución Conocida

Antigua provincia de las Villas; Managua en Provincia de la Habana.

Luis Lazo; Pan de Guajaibón;

La Habana, Ciudad de la Habana, Matanzas, Santiago de Cuba; América del Sur; América Central

Provincias de la Habana y Matanzas

No registrada en el catálogo
Sierra el Queque; Sierra del Abra

Mogote el Queque

Sierra de Ancón; Sitio del Infierno; Sierra de San Vicente; Mogote el Queque.

Hoyos de San Antonio

Sierra del Medio

Mogote La Feita

Mogote Jagua Vieja en Viñales; Mogote Pico; Mogote Bella María

En todas las localidades muestreadas

Distribución Ampliada

Abra del Café en Sierra del Grillo

Mogote Cayos de San Felipe

Sierra Pan de Azúcar

Mogotes Zacarías, Coco Solo; Sierras la Caoba, Viñales, Guasasa, Pan de Azúcar

En todas las localidades muestreadas 
CUADRO 4 (Continuación)

Nuevos registro

TABLE 4 (Continued)

New records

\begin{tabular}{|c|c|c|}
\hline Especie & Distribución conocida & Distribución ampliada \\
\hline Eurycampta pinarensis & Luis Lazo & $\begin{array}{l}\text { En todas las localidades muestreadas, } \\
\text { excepto los mogotes Dopico y La Feita }\end{array}$ \\
\hline Emoda sagraiana* & Pan de Azúcar & En todas las localidades muestreadas \\
\hline Annularops coronadoi* & $\begin{array}{l}\text { Hoyo de Jaruco, Costanera de San Vicente, } \\
\text { El Queque, Laguna de Piedra }\end{array}$ & $\begin{array}{l}\text { Mogotes Irene, Pan de Azúcar, Coco } \\
\text { Solo; Sierras Pan de Azúcar, Viñales, } \\
\text { Guasasa, Tumbadero }\end{array}$ \\
\hline Rhytidothyra bilabiada* & $\begin{array}{l}\text { Sierra del Infierno, Kilómetro } 14 \text { entre Pinar } \\
\text { del Río y Viñales, Pan de Azúcar, Costanera, } \\
\text { Cayos d San Felipe, Laguna de Piedra }\end{array}$ & En todas las localidades muestreadas \\
\hline Tomelasmus irroratus* & $\begin{array}{l}\text { Sitio del Infierno, mogote Pan de Azúcar, } \\
\text { oeste de la Sierra de La Chorrera }\end{array}$ & $\begin{array}{l}\text { En todas las localidades muestreadas, } \\
\text { excepto los mogotes La Feita e Irene, y la } \\
\text { Sierra Pan de Azúcar }\end{array}$ \\
\hline Geminicoptis terebella* & Mogote del Mármol & $\begin{array}{l}\text { Mogotes Pan de Azúcar, Zacarías; } \\
\text { Sierras La Caoba, Viñales, del Quemado, } \\
\text { Guasasa, Pan de Azúcar }\end{array}$ \\
\hline
\end{tabular}

* Especies con distribución más bien generalizada.

* species with distribution rather than wide.

CUADRO 5

Especies no encontradas en los muestreos

TABLE 5

Species not found in this sampling

$\begin{array}{ll}\text { Especie } & \text { Localidad en la que no se observó durante el muestreo } \\ \text { Semitrochatella alboviridis } & \text { Dos Hermanos } \\ \text { Farcimen hendersoni } & \text { Mogote Pan de Azúcar } \\ \text { Annularops tryoni } & \text { Sierra Viñales } \\ \text { Chondropometes eximium } & \text { Sierra del Quemado } \\ \text { Eutudorops troscheli } & \text { Pan de Azúcar } \\ \text { Hendersonina echinulata } & \text { Viñales, sin precisar localidades; no se ha vuelto a recolectar } \\ \text { Hendersonina deceptor } & \text { Sierra de Viñales } \\ \text { Nodulia oblita } & \text { Pan de Azúcar } \\ \text { Tomelasmus azucarensis } & \text { Pan de Azúcar } \\ \text { Tomelasmus hesperius } & \text { Sierra Viñales } \\ \text { Oleacina incisa } & \text { Distribución generalizada hacia el occidente de Cuba } \\ \text { Jeanneretia modica } & \text { Región de Viñales }\end{array}$


y a las colecciones consultadas debieron estar presentes en alguna de las localidades muestreadas y de las que, sin embargo, no se observaron ni siquiera sus conchas vacías

\section{DISCUSIÓN}

Las 136 especies de moluscos terrestres detectadas en la región de Viñales representan un valor elevado de riqueza de especies en el contexto mundial. Este valor es inferior al observado en algunas otras regiones del mundo, seguramente de mayor área, como el archipiélago hawaiano en el que se distribuyen 750 especies (Cowie 1995) y los estados mexicanos de Veracruz y Texas en los que se han registrado 242 y 148 especies respectivamente (Correa-Sandoval \& Salazar 2005), pero superior a las de otros estados mexicanos como Nuevo León (86), Tamaulipas (105), San Luis Potosí (96), así como a las 51 especies del noreste de Yorkshire en Inglaterra (Wardhaugh 1996), a las 82 especies del sur de la península de Michigan (Nekola 2004), y a las 84 especies provenientes de los bosques de Tanzania (Tattersfield et al. 2006). Hasta el momento, el de Viñales es el mayor valor de riqueza de especies detectado a escala regional en el archipiélago cubano, superior a las 86 especies de moluscos terrestres observadas por Jaume (1972) en la región de Sierra del Rosario. No obstante, es posible que estudios futuros en otras regiones de la cordillera de Guanigüanico, como Sumidero, en la que existe mayor proporción de sierra cárstica que en Viñales (Luis 2000), revele valores superiores de riqueza malacológica.

Esta elevada riqueza de especies puede estar favorecida por las condiciones climáticas, fundamentalmente de humedad, la disponibilidad de carbonato de calcio y la diversidad de plantas y hongos comestibles, que propician las condiciones para que se desarrollen poblaciones viables de moluscos terrestres. Todo esto, unido a eventos de especiación especialmente favorecidos por la poca vagilidad de los moluscos y su distribución en un relieve de elevaciones cársticas separadas por valles, puede haber contribuido a generar tanto la diversidad como el elevado endemismo malacológico que caracteriza a la región.

La alta representación de las familias Annulariidae y Urocoptidae era de esperar, debido a que el $69 \%$ de las especies de moluscos terrestres de Cuba listadas por Espinosa y Ortea (1999) pertenecen a alguna de estas dos familias. Sin embargo, aunque Urocoptidae es la familia de gasterópodos terrestres cubanos con mayor número de especies (480), en este estudio se ha detectado mayor número de especies en la familia Annularidae. Esto podría estar condicionado no porque realmente existan más especies de la familia Annularidae distribuidas en Viñales, sino por la insuficiencia de las tres fuentes consultadas, atribuible fundamentalmente a las dificultades para detectar las especies de pequeño tamaño, frecuentes en la familia Urocoptidae, unido a los impedimentos para acceder a muchas áreas de las localidades de Viñales debido a las características del relieve. Probablemente otras dificultades también han estado presentes para detectar y recolectar ejemplares de otras familias y géneros de gasterópodos terrestres, pues no existe una correspondencia total en la composición de especies detectada según las tres fuentes abordadas.

Los nuevos registros de localidades (Cuadro 4) deben tenerse en cuenta durante los análisis del endemismo y de los patrones de distribución en los moluscos terrestres de Viñales y de Cuba en general. Las cinco especies no autóctonas detectadas, Bradybaena similaris, Zachrysia auricoma, Streptostele musaecola, Subulina octona, Leidyula floridana, presentan según la literatura una distribución generalizada por el territorio nacional o su región occidental y podrían tenerse presente también durante futuros estudios como especies indicadoras de antropización de las elevaciones cársticas de Viñales y de su parque nacional en particular, pues parece haber una tendencia a que estas especies extiendan su distribución de las áreas periurbanas hacia las elevaciones cársticas. De hecho, ninguna de estas especies sinantrópicas había sido detectada por los coleccionistas de décadas anteriores, que posiblemente limitaban 
sus recolectas a las elevaciones cársticas, y sin embargo en los muestreos efectuados durante este estudio se han observado en numerosas localidades.

Debido a que sólo se muestreó en 12 localidades, el 13\% (15 especies) de endemismo local que caracteriza a los moluscos terrestres de la región probablemente ha podido condicionar en parte que sólo se hayan observado la mitad de las especies registradas para Viñales. También es posible que en la malacofauna de Viñales se estén manifestando casos de extinciones locales. De hecho, Espinosa y Ortea (1999) sugirieron la posibilidad de que las especies Chondrothyra percrassa y Hendersonina echinulata, distribuidas también en elevaciones cársticas, se hayan extinguido, y Vales et al. (1998) incluyeron otras 131 especies de moluscos terrestres de Cuba en alguna de las categorías de amenaza establecidas internacionalmente.

La desaparición de las poblaciones locales de las 12 especies reflejadas en el Cuadro 5, incluyendo las posibles extinciones definitivas de las especies Tomelasmus azucarensis y Nodulia oblita, puede que esté asociada a la transformaciones antrópicas que se han evidenciado, según Luis (2000), durante décadas anteriores en las elevaciones cársticas de Viñales y que a su vez probablemente han condicionado la aparición de las cinco especies de moluscos terrestres sinantrópicas, que en años anteriores sólo limitaban su distribución a jardines y áreas agrícolas de Viñales. Es posible que la influencia humana esté condicionando el empobrecimiento de la diversidad malacológica, aspecto corroborado por Berovides et al (1994) en zonas de Sierra de los Órganos más influenciadas por la agricultura y el turismo. En general, se ha detectado una declinación a nivel mundial en la diversidad de especies de moluscos no marinos (Lydeard et al. 2004), que en casos como los de las islas del Pacífico están asociados a la destrucción del hábitat y al impacto de especies invasoras (Cowie \& Robinson 2003), y en otros como los de Malasia se relacionan con la agricultura, la tala, los incendios y extracción de rocas
(Schilthuizen et al. 2005) o el alpinismo como en Ontario (McMillan et al. 2003). Todo lo cual confirma la necesidad urgente de desarrollar bases de datos y monitoreos poblacionales que se tengan en cuenta durante la elaboración de planes de manejo para el Parque Nacional Viñales con fines de conservación.

Las poblaciones de algunas de las especies endémicas de Viñales están desapareciendo y las de moluscos terrestres no autóctonos amplían su distribución hacia las elevaciones cársticas de la región. El conocimiento del área de distribución de sus especies es todavía inexacto. Se necesita aprovechar la información disponible en las colecciones malacológicas del país, facilitar el acceso a las monografías malacológicas y describir los patrones a través de los cuales se estructuran sus comunidades de moluscos terrestres, para contribuir no solamente al conocimiento de este importante filo sino también poder desarrollar acciones de manejo que contribuyan a la restauración y conservación de una de las comunidades con mayor diversidad y endemismos de moluscos terrestres de Cuba, y posiblemente del mundo.

\section{AGRADECIMIENTOS}

La presente publicación forma parte de los proyectos "Incremento, conservación y manejo de las Colecciones Zoológicas" y "Especies y colecciones biológicas: sistemática y taxonomía de algunos grupos representativos de la biodiversidad cubana. Un estudio sobre comunidades de moluscos terrestres de las elevaciones cársticas de la región de Viñales", desarrollados por el Instituto de Ecología y Sistemática (IES) de Cuba y financiados por la Agencia de Ambiente del Ministerio de Ciencia, Tecnología y Ambiente de Cuba. Este artículo ha sido parcialmente financiado por el Ministerio de Educación y Ciencia de España y por el FEDER (Unión Europea) mediante el proyecto CGL2006-09567/BOS.

\section{RESUMEN}

De acuerdo a los muestreos efectuados en localidades representativas de las elevaciones cársticas de Viñales 
(Cuba), la consulta de catálogos no automatizados de colecciones malacológicas y la revisión de la literatura, se dan a conocer 136 especies, agrupadas en 49 géneros y 19 familias, de moluscos terrestres procedentes de la región. El 90\% de las especies identificadas son endémicas de Cuba y sólo cinco especies no son autóctonas del archipiélago cubano. Las familias mejor representadas son Annulariidae y Urocoptidae. Los géneros con mayor número de especies son Chondrothyra y Liocallonia. Cuarenta y cuatro especies detectadas en Viñales no estaban representadas en las colecciones consultadas, y 23 de ellas han sido recolectadas en este trabajo y aportadas a las colecciones del Instituto de Ecología y Sistemática de Cuba. Se dan a conocer 16 nuevos registros de especies en localidades de la región, mientras que 12 especies parecen haberse extinguido localmente.

Palabras clave: gasterópodos, colecciones malacológicas, diversidad de especies, distribución, Viñales, Cuba.

\section{REFERENCIAS}

Abbott, R.T. 1989. Compendium of landshells. American Malacologists, Inc. Melbourne, Florida, EEUU.

Berovides V., R. Tadeo \& M. Alfonso. 1994. Concluye el estudio sobre la malacocenosis de la Sierra de los Órganos, Pinar del Río. Cocuyo 1: 7-8.

Correa-Sandoval, A. \& M.C. Salazar. 2005. Gasterópodos terrestres del sur de Nuevo León, México. Acta. Zool. Mex. 21: 51-61.

Cowie, R.H. 1995. Variation in species diversity and shell shape in Hawaian land snails: in situ speciation and ecological relationships. Evolution 49: 1191-1202.

Cowie R.H. \& A.C. Robinson 2003. The decline of native Pacific island faunas: changes in status of the land snails of Samoa through the 20th century. Biol. Conserv. 1: 55-65

Emberton K.C., T. Pearce \& R. Randalana. 1996. Quantitatively sampling land-snail species richness in Madagascan Rainforests. Malacologia 38: 203-212.

Emberton K.C., T. Pearce, P.F. Kasigwa, P. Tattersfield \& Z. Habibu. 1997. High diversity and regional endemism in land snails of eastern Tanzania. Biodivers. Conserv. 2: 1123-1136.

Espinosa, J \& J. Ortea. 1999. Moluscos terrestres del archipiélago cubano. Avicenia. suplemento 2: 1-137.

Espinosa, J., J. Ortea \& A. Valdés. 1994. Clasificación taxonómica y endemismos de los moluscos terrestres de Cuba. Avicenia. 1: 111-124.
Jaume, M.L. 1972. Lista de los moluscos de la Sierra del Rosario. La Habana. Serie. Biológica. 41:1-29.

Landeiro, E. 2003. Análisis de la estructura de los paisajes del parque nacional Viñales. Tesis en opción al grado científico de Licenciado en Ciencias Geográficas. Universidad de la Habana, Habana, Cuba.

Luis, M. 2001. Evaluación para la protección de los mogotes de la Sierra de los Órganos y el Pan de Güajaibón. Tesis en opción al grado científico de Doctor en Ciencias Geográficas. Universidad de la Habana, Habana, Cuba.

Lydeard, C., R.H. Cowie, W.F. Ponder, A.E. Bogan, P. Bouchet, S.A. Clark, K.S. Cummings, T.J. Frest, O.G. Gargominy, D.G. Herbert, R. Hershler, K.E. Perez, B.R. Seddom, E.E. Strong, K.E. Roth, B. Seddon, M. Strong \& F.G. Thompson. 2004. The global decline of nonmarine mollusks. BioScience 54: 321-330.

McMillan, M.A., J.C Nekola \& D.W. Larson. 2003. Effects of rock climbing on the land snail community of the Niagara Escarpment in southern Ontario, Canada. Conserv. Biol. 17: 616-621

Nekola, J.C. 2004. Terrestrial gastropod fauna of northeastern Wisconsin and the southern Upper Peninsula of Michigan. Amer. Malacol. Bull. 18: 21-44.

Nuñez, A., N. Viña, M. Acevedo, J. Mateo, M. Iturralde \& A. Graña. 1984. Cuevas y Carsos. Editorial Científico Técnica, La Habana, Cuba.

Pérez, A.M., J.C. Vilaseca \& N. Ziane. 1996. Sinecología básica de moluscos terrestres en cuatro formaciones vegetales de Cuba. Rev. Biol. Trop. 44: 133-146.

Programa 21. 1992. Informe de la conferencia de las Naciones Unidas sobre Ambiente y Desarrollo. Editorial Academia, La Habana, Cuba.

Ruppert, E.E. \& R.D. Barnes. 1996. Zoología de los invertebrados. McGraw-Hill interamericana, México D.F., México.

Schilthuizen, M., T.S. Liew, B.B Elahan \& I. LackmanAncrenaz. 2005. Effects of karst forest degradation on pulmonate and prosobranch land snail communities in Sabah, Malaysian Borneo. Conserv. Biol. 19: 949-954.

Tattersfield, P. 1996. Local patterns of land snail diversity in a Kenyan rain forest. Malacologia 38: 161-180.

Tattersfield, P., M.B. Seddon, C. Ngereza \& B. Rowson. 2006. Elevational variation in diversity and composition of land-snail faunas in a Tanzanian forest. African J. Ecol. 44: 47-60. 
Torre de la, C. \& P. Bartsch. 1938. The Cuban Operculate Land Shells of the Subfamily Chondropominae. Proc. U.S. Nat. Mus. 85: 193-403.

Vales, M., A. Álvarez., L. Montes \& A. Ávila. 1998. Estudio Nacional sobre la Diversidad Biológica en la República de Cuba. CESYTA, Madrid, España.

Vilamajó, D., M.A. Vales, R.P. Capote, D. Salabarria \& J.M. Menéndez. 2002. Estrategia Nacional para la
Diversidad Biológica y Plan de Acción en la República de Cuba. Editorial Academia, La Habana, Cuba.

Wardhaugh, A.A. 1996. The terrestrial molluscan fauna of some woodlands in north east Yorkshire, England. J. Conchology. 35: 313-327

Winter, A.J \& E. Gittenberger. 1998. The land snail fauna of square kilometer patch of rainforest in southestern Cameroon. Malacologia 40: 231-250. 